\title{
New Hybrid Steepest Descent Algorithms for Equilibrium Problem and Infinitely Many Strict Pseudo-Contractions in Hilbert Spaces
}

\author{
Peichao Duan \\ College of Science, Civil Aviation University of China, Tianjin 300300, China \\ Correspondence should be addressed to Peichao Duan; pcduancauc@126.com
}

Received 4 May 2013; Accepted 17 June 2013

Academic Editor: Gue Myung Lee

Copyright (C) 2013 Peichao Duan. This is an open access article distributed under the Creative Commons Attribution License, which permits unrestricted use, distribution, and reproduction in any medium, provided the original work is properly cited.

We propose an explicit iterative scheme for finding a common element of the set of fixed points of infinitely many strict pseudocontractive mappings and the set of solutions of an equilibrium problem by the general iterative method, which solves the variational inequality. In the setting of real Hilbert spaces, strong convergence theorems are proved. The results presented in this paper improve and extend the corresponding results reported by some authors recently. Furthermore, two numerical examples are given to demonstrate the effectiveness of our iterative scheme.

\section{Introduction}

Let $H$ be a real Hilbert space with inner product $\langle$,$\rangle and$ induced norm $\|\cdot\|$. Let $C$ be a nonempty closed convex subset of $H$.

Let $A: C \rightarrow H$ be a nonlinear mapping; we consider the problem of finding $x^{*} \in C$ such that

$$
\left\langle A x^{*}, x-x^{*}\right\rangle \geq 0, \quad \forall x \in C .
$$

It is known as the variational inequality problem (denoted by $\mathrm{VI}(A, C))$.

Generally, $A$ is assumed to be Lipschitzian and strongly monotone. The relative definitions are listed as follows.

(i) $A$ is called $k$-Lipschitzian on $C$, if there exists a constant $k>0$ such that

$$
\|A x-A y\| \leq k\|x-y\|, \quad \forall x, y \in C .
$$

(ii) $A$ is said to be $\eta$-strongly monotone on $C$, if there exists a constant $\eta>0$ such that

$$
\langle A x-A y, x-y\rangle \geq \eta\|x-y\|^{2}, \quad \forall x, y \in C .
$$

(iii) A mapping $S$ of $C$ is said to be a $\kappa$-strict pseudocontraction if there exists a constant $\kappa \in[0,1)$ such that

$$
\begin{aligned}
& \|S x-S y\|^{2} \leq\|x-y\|^{2}+\kappa\|(I-S) x-(I-S) y\|^{2} \\
& \quad \text { for all } x, y \in C \text {; see [1]. }
\end{aligned}
$$

(iv) A mapping $S$ of $C$ is said to be a nonexpansive mapping if it is strictly pseudo-contractive with constant $\kappa=0$.

Obviously, the class of strict pseudo-contractions strictly includes the class of nonexpansive mappings. We denote the set of fixed points of $S$ by $F_{i x}(S)$ (i.e., $F_{i x}(S)=\{x \in C: S x=$ $x\})$.

Let $F$ be a bifunction from $C \times C$ to $\mathbb{R}$, where $\mathbb{R}$ is the set of real numbers.

The equilibrium problem for $F: C \times C \rightarrow \mathbb{R}$ is to determine its equilibrium points, that is, the set

$$
\{x \in C: F(x, y) \geq 0, \forall y \in C\} .
$$

The set of such solutions is denoted by $\mathrm{EP}(F)$.

Many problems in applied sciences such as physics, optimization, and economics reduce into finding some element of $\mathrm{EP}(F)$. Some methods have been proposed to solve the 
equilibrium problem (5); see, for instance, [2-6]. In particular, Combettes and Hirstoaga [7] proposed several methods for solving the equilibrium problem. On the other hand, Mann [8] and Shimoji and Takahashi [9] considered iterative schemes for finding a fixed point of a nonexpansive mapping. Further, Acedo and $\mathrm{Xu}$ [10] projected new iterative methods for finding a fixed point of strict pseudo-contractions.

In 2006, Marino and Xu [5] proposed a general iterative method and proved that the algorithm converged strongly. Recently, Tian [11] revealed the inner contact of Yamada's algorithm [12] and viscosity iterative algorithm and then introduced a new general iterative algorithm combining a $k$-Lipschitzian and $\eta$-strong monotone operator. On this basis, Wang [13] considered a general composite iterative method for infinitely many strict pseudo-contractions in 2010. However, the $W$-mapping used in Wang's paper requires many composite operations. Very recently, He and Sun [14] proposed a new operator $L_{n}$ to replace the $W$-mapping for infinite family nonexpansive mappings.

The mapping $L_{n}$ is defined as follows:

$$
L_{n}=\sum_{i=1}^{n} \frac{\omega_{i}}{s_{n}} T_{i} \quad(n=1,2, \ldots),
$$

where $\left\{\omega_{i}\right\} \subset(0,1)$ such that $\sum_{i=1}^{\infty} \omega_{i}=1, s_{n}=\sum_{i=1}^{n} \omega_{i}$, and $\left\{T_{i}\right\}$ are infinite nonexpansive mappings. Because it does not contain many composite computations, it is more simple and easy to realize.

In this paper, we combine the operator $L_{n}$ and the general iterative algorithm to propose a new explicit iterative scheme involving equilibrium problem (5) and an infinite family of strict pseudo-contractions. Under certain assumptions, we will prove that the sequence converges strongly. Further an example will be given to demonstrate the effectiveness of our iterative scheme and another will be given to compare numerical results and convergence rate of the algorithm in this paper and [15].

\section{Preliminaries}

In the sequel, we will make use of the following lemmas in a real Hilbert space $H$.

Lemma 1. Let $H$ be a real Hilbert space. There hold the following identities:

(i)

$$
\|x-y\|^{2}=\|x\|^{2}-\|y\|^{2}-2\langle x-y, y\rangle, \quad \forall x, y \in H,
$$

(ii)

$$
\begin{aligned}
& \|t x+(1-t) y\|^{2} \\
& =t\|x\|^{2}+(1-t)\|y\|^{2}-t(1-t)\|x-y\|^{2}, \\
& \forall t \in[0,1], \quad \forall x, y \in H .
\end{aligned}
$$

Lemma 2 (see [13]). Let $A: H \rightarrow H$ be a $k$-Lipschitzian and $\eta$-strongly monotone operator on a Hilbert space $H$ with $k>0$, $\eta>0,0<\mu<2 \eta / k^{2}$, and $0<t<1$. Then $S=(I-t \mu A):$ $H \rightarrow H$ is a contraction with contractive coefficient $1-t \tau$ and $\tau=\mu\left(2 \eta-\left(\mu k^{2} / 2\right)\right)$.

Lemma 3 (see [1]). Let $S: C \rightarrow C$ be a $\kappa$-strict pseudocontraction. Define $T: C \rightarrow C$ by $T x=\lambda x+(1-\lambda) S x$ for each $x \in C$. Then, as $\lambda \in[\kappa, 1), T$ is a nonexpansive mapping such that $F_{i x}(T)=F_{i x}(S)$.

Lemma 4. Let $V: C \rightarrow H$ be an l-Lipschitz mapping with coefficient $l \geq 0$ and $A: C \rightarrow H$ ak-Lipschitzian continuous operator and $\eta$-strongly monotone operator with $k>0, \eta>0$. Then, for $0<\gamma<\mu \eta / l$,

$$
\begin{gathered}
\langle x-y,(\mu A-\gamma V) x-(\mu A-\gamma V) y\rangle \\
\geq(\mu \eta-\gamma l)\|x-y\|^{2}, \quad x, y \in H .
\end{gathered}
$$

That is, $\mu A-\gamma V$ is strongly monotone with coefficient $\mu \eta-\gamma l$.

Proof. Since $A$ is $l$-Lipschitz and $\eta$-strongly monotone, it is easy to get

$$
\begin{aligned}
& \langle x-y,(\mu A-\gamma V) x-(\mu A-\gamma V) y\rangle \\
& \quad=\mu\langle x-y, A x-A y\rangle-\gamma\langle x-y, V x-V y\rangle \\
& \quad \geq(\mu \eta-\gamma l)\|x-y\|^{2}, \quad x, y \in C .
\end{aligned}
$$

Lemma 5 (see [16]). Assume that $\left\{a_{n}\right\}$ is a sequence of nonnegative real numbers such that

$$
a_{n+1} \leq\left(1-\gamma_{n}\right) a_{n}+\delta_{n},
$$

where $\left\{\gamma_{n}\right\}$ is a sequence in $(0,1)$ and $\left\{\delta_{n}\right\}$ is a sequence such that

(i)

$$
\sum_{n=1}^{\infty} \gamma_{n}=\infty
$$

(ii)

$$
\limsup _{n \rightarrow \infty} \frac{\delta_{n}}{\gamma_{n}} \leq 0 \text { or } \sum_{n=1}^{\infty}\left|\delta_{n}\right|<\infty \text {. }
$$

Then, $\lim _{n \rightarrow \infty} a_{n}=0$.

Let $\left\{S_{n}\right\}$ be a sequence of $\kappa_{n}$-strict pseudo-contractions. Define $S_{n}^{\prime}=\theta_{n} I+\left(1-\theta_{n}\right) S_{n}, \theta_{n} \in\left[\kappa_{n}, 1\right)$. Then, by Lemma 3, $S_{n}^{\prime}$ is nonexpansive. In order to find the common fixed point set of infinite mappings, $W$-mapping is often used; see $[9,13$, $15,17,18]$ and references therein. The mapping $W_{n}$ is defined by

$$
\begin{gathered}
U_{n, n+1}=I, \\
U_{n, n}=t_{n} S_{n}^{\prime} U_{n, n+1}+\left(1-t_{n}\right) I, \\
U_{n, n-1}=t_{n-1} S_{n-1}^{\prime} U_{n, n}+\left(1-t_{n-1}\right) I,
\end{gathered}
$$




$$
\begin{gathered}
U_{n, i}=t_{i} S_{i}^{\prime} U_{n, i+1}+\left(1-t_{i}\right) I, \\
\vdots \\
U_{n, 2}=t_{2} S_{2}^{\prime} U_{n, 3}+\left(1-t_{2}\right) I, \\
W_{n}=U_{n, 1}=t_{1} S_{1}^{\prime} U_{n, 2}+\left(1-t_{1}\right) I,
\end{gathered}
$$

where $t_{1}, t_{2}, \ldots$ are real numbers such that $0 \leq t_{n}<1$. Such a mapping $W_{n}$ is called a $W$-mapping generated by $S_{1}^{\prime}, S_{2}^{\prime}, \ldots$ and $t_{1}, t_{2}, \ldots$. As we have seen, $W$-mapping contains many composite computation of $S_{n}^{\prime}$, and it is complicated and needs a large number of complex operations. In [14], He and Sun proposed a new hybrid steepest descent method for solving fixed point problem defined on the common fixed point set of infinite nonexpansive mappings.

Lemma 6 (see [14]). Let $H$ be a real Hilbert and $T_{i}: H \rightarrow H$ $(i=1,2, \ldots)$ all nonexpansive mappings with $\bigcap_{i=1}^{\infty} F_{i x}\left(T_{i}\right) \neq \emptyset$. Let $T=\sum_{i=1}^{\infty} \omega_{i} T_{i}(i=1,2, \ldots)$, where $\left\{\omega_{i}\right\} \subset(0,1)$ such that $\sum_{i=1}^{\infty} \omega_{i}=1$. Then $T$ is a nonexpansive mapping with $F_{i x}(T)=$ $\bigcap_{i=1}^{\infty} F_{i x}\left(T_{i}\right)$.

Lemma 7 (see [14]). Let $H$ be a real Hilbert and $T_{i}: H \rightarrow H$ $(i=1,2, \ldots)$ all nonexpansive mappings with $\bigcap_{i=1}^{\infty} F_{i x}\left(T_{i}\right) \neq \emptyset$. Let $T=\sum_{i=1}^{\infty} \omega_{i} T_{i}$, where $\left\{\omega_{i}\right\} \subset(0,1)$ such that $\sum_{i=1}^{\infty} \omega_{i}=$ 1. Assume $L_{n}=\sum_{i=1}^{n} \omega_{i} T_{i} / s_{n}$, where $s_{n}=\sum_{i=1}^{n} \omega_{i}$. Then $L_{n}$ uniformly converges to $T$ in each bounded subset in $H$.

For solving the equilibrium problem, let us assume that the bifunction $F$ satisfies the following conditions:

(A1) $F(x, x)=0$ for all $x \in C$;

(A2) $F$ is monotone; that is, $F(x, y)+F(y, x) \leq 0$ for any $x, y \in C$;

(A3) for each $x, y, z \in C, \quad \lim \sup _{t \rightarrow 0} F(t z+(1-t) x, y) \leq$ $F(x, y)$;

(A4) $F(x, \cdot)$ is convex and lower semicontinuous for each $x \in C$.

We recall some lemmas which will be needed in the rest of this paper.

Lemma 8 (see [2]). Let $C$ be a nonempty closed convex subset of $H$, let $F$ be bifunction from $C \times C$ to $\mathbb{R}$ satisfying (A1)-(A4), and let $r>0$ and $x \in H$. Then there exists $z \in C$ such that

$$
F(z, y)+\frac{1}{r}\langle y-z, z-x\rangle \geq 0, \quad \forall y \in C .
$$

Lemma 9 (see [7]). For $r>0, x \in H$, define a mapping $T_{r}$ : $H \rightarrow C$ as follows:

$$
\begin{aligned}
& T_{r}(x) \\
& \quad=\left\{z \in C \mid F(z, y)+\frac{1}{r}\langle y-z, z-x\rangle \geq 0, \forall y \in C\right\}
\end{aligned}
$$

for all $x \in H$. Then, the following statements hold: (i) $T_{r}$ is single valued;

(ii) $T_{r}$ is firmly nonexpansive; that is, for any $x, y \in H$,

$$
\left\|T_{r} x-T_{r} y\right\|^{2} \leq\left\langle T_{r} x-T_{r} y, x-y\right\rangle ;
$$

(iii) $F_{i x}\left(T_{r}\right)=\mathrm{EP}(F)$;

(iv) $\mathrm{EP}(F)$ is closed and convex.

Lemma 10 (see [19]). Let $\left\{x_{n}\right\}$ and $\left\{z_{n}\right\}$ be bounded sequences in a Banach space and $\left\{\beta_{n}\right\}$ a sequence of real numbers such that $0<\liminf _{n \rightarrow \infty} \beta_{n} \leq \limsup _{n \rightarrow \infty} \beta_{n}<1$ for all $n=$ $0,1,2, \ldots$ Suppose that $x_{n+1}=\left(1-\beta_{n}\right) z_{n}+\beta_{n} x_{n}$ for all $n=$ $0,1,2, \ldots$ and $\lim \sup _{n \rightarrow \infty}\left\|z_{n+1}-z_{n}\right\|-\left\|x_{n+1}-x_{n}\right\| \leq 0$. Then $\lim _{n \rightarrow \infty}\left\|z_{n}-x_{n}\right\|=0$.

Lemma 11 (see [6]). Let C, H, F, and $T_{r} x$ be as in Lemma 9. Then the following holds:

$$
\left\|T_{s} x-T_{t} x\right\|^{2} \leq \frac{s-t}{s}\left\langle T_{s} x-T_{t} x, T_{s} x-x\right\rangle
$$

for all $s, t>0$ and $x \in H$.

Lemma 12 (see [13]). Let $H$ be a Hilbert space, $C$ a nonempty closed convex subset of $H$, and $T: C \rightarrow C$ a nonexpansive mapping with $F_{i x}(T) \neq \emptyset$. If $\left\{x_{n}\right\}$ is a sequence in $C$ weakly converging to $x$ and if $\left\{(I-T) x_{n}\right\}$ converges strongly to $y$, then $(I-T) x=y$.

We adopt the following notations:

(1) $x_{n} \rightarrow x$ stands for the weak convergence of $\left\{x_{n}\right\}$ to $x$,

(2) $x_{n} \rightarrow x$ stands for the strong convergence of $\left\{x_{n}\right\}$ to $x$.

\section{Main Result}

Recall that, given a nonempty closed convex subset $C$ of a real Hilbert space $H$, for any $x \in H$, there exists a unique nearest point in $C$, denoted by $P_{C} x$, such that

$$
\left\|x-P_{C} x\right\| \leq\|x-y\|
$$

for all $y \in C$. Such a $P_{C}$ is called the metric (or the nearest point) projection of $H$ onto $C$. As we all know, $y=P_{C} x$ if and only if there holds the following relation:

$$
\langle x-y, y-z\rangle \geq 0 \quad \forall z \in C .
$$

Throughout the rest of this paper, we always assume that $V$ is an $l$-Lipschitzian mapping of $H$ into itself with coefficient $l \geq 0$ and $A$ is a $k$-Lipschitzian continuous operator and $\eta$ strongly monotone on $H$ with $k>0, \eta>0$. Assume that $0<\mu<2 \eta / k^{2}$ and $0<\gamma<\mu\left(\eta-\left(\mu k^{2} / 2\right)\right) / \alpha=\tau / l$.

Define a mapping $U_{n}=\beta_{n} I+\left(1-\beta_{n}\right) L_{n} T_{r_{n}}$. Since both $L_{n}$ and $T_{r_{n}}$ are nonexpansive, it is easy to get that $U_{n}$ is also nonexpansive. Consider the mapping $G_{n}$ on $H$ defined by

$$
G_{n} x=\alpha_{n} \gamma V(x)+\left(I-\alpha_{n} \mu A\right) U_{n} x, \quad \forall x \in H, n \in N,
$$


where $\alpha_{n} \in(0,1)$. By Lemmas 2 and 9, we have

$$
\begin{aligned}
\left\|G_{n} x-G_{n} y\right\| & \leq \alpha_{n} \gamma\|V x-V y\|+\left(1-\alpha_{n} \tau\right)\left\|U_{n} x-U_{n} y\right\| \\
& \leq \alpha_{n} \gamma l\|x-y\|+\left(1-\alpha_{n} \tau\right)\|x-y\| \\
& =\left(1-\alpha_{n}(\tau-\gamma l)\right)\|x-y\| .
\end{aligned}
$$

Since $0<1-\alpha_{n}(\tau-\gamma l)<1$, it follows that $G_{n}$ is a contraction. Therefore, by the Banach contraction principle, $G_{n}$ has a unique fixed point $x_{n}^{V} \in H$ such that

$$
x_{n}^{V}=\alpha_{n} \gamma V\left(x_{n}^{V}\right)+\left(I-\alpha_{n} \mu A\right) U_{n} x_{n}^{V} .
$$

For simplicity, we will write $x_{n}$ for $x_{n}^{V}$ provided no confusion occurs. Next we prove the sequence $\left\{x_{n}\right\}$ converges strongly to a $x^{*} \in \Omega=\bigcap_{i=1}^{\infty} F_{i x}\left(S_{i}\right) \cap \mathrm{EP}(F)$ which solves the variational inequality

$$
\left\langle(\gamma V-\mu A) x^{*}, p-x^{*}\right\rangle \leq 0, \quad \forall p \in \Omega .
$$

By the property of the projection, we can get $x^{*}=P_{\Omega}(I-\mu A+$ $\gamma V) x^{*}$ equivalently.

Theorem 13. Let $C$ be a nonempty closed convex subset of a real Hilbert space $H$ and $F$ a bifunction from $C \times C$ to $\mathbb{R}$ satisfying (A1)-(A4). Let $S_{i}: C \rightarrow C$ be family $\kappa_{i}$-strict pseudo-contractions for some $0 \leq \kappa_{i}<1$. Assume the set $\Omega=\bigcap_{i=1}^{\infty} F\left(S_{i}\right) \cap \mathrm{EP}(F) \neq \emptyset$. Let $V$ be an l-Lipschitzian mapping of $H$ into itself with $l \geq 0$, and let $A$ be a $k$-Lipschitzian continuous operator and $\eta$-strongly monotone on $H$ with $k>0$, $\eta>0,0<\mu<2 \eta / k^{2}$, and $0<\gamma<\mu\left(\eta-\left(\mu k^{2} / 2\right)\right) / l=\tau / l$. For every $n \in \mathbb{N}$, let $L_{n}$ be the mapping generated by $S_{i}^{\prime}$ and $0<$ $\omega_{i}<1$ with $\sum_{i=1}^{\infty} \omega_{i}=1$ according to (6). Given $x_{1} \in H$, let $\left\{x_{n}\right\}$ and $\left\{u_{n}\right\}$ be sequences generated by the following algorithm:

$$
\begin{gathered}
u_{n}=T_{r_{n}} x_{n}, \\
y_{n}=\beta_{n} x_{n}+\left(1-\beta_{n}\right) L_{n} u_{n}, \\
x_{n+1}=\alpha_{n} \gamma V x_{n}+\left(I-\mu \alpha_{n} A\right) y_{n} .
\end{gathered}
$$

If $\left\{\alpha_{n}\right\},\left\{\beta_{n}\right\}$, and $\left\{r_{n}\right\}$ satisfy the following conditions:

(i) $\left\{\alpha_{n}\right\} \subset(0,1), \lim _{n \rightarrow \infty} \alpha_{n}=0$, and $\sum_{n=1}^{\infty} \alpha_{n}=\infty$;

(ii) $0<\liminf _{n \rightarrow \infty} \beta_{n} \leq \lim \sup _{n \rightarrow \infty} \beta_{n}<1$;

(iii) $\left\{r_{n}\right\} \subset(0, \infty), \liminf _{n \rightarrow \infty} r_{n}>0$, and $\lim _{n \rightarrow \infty} \mid r_{n+1}-$ $r_{n} \mid=0$,

then, $\left\{x_{n}\right\}$ converges strongly to $x^{*} \in \Omega$, which solves the variational inequality (24).

Proof. The proof is divided into several steps.

Step 1. Show first that $\left\{x_{n}\right\}$ is bounded.

Taking any $p \in \Omega$, by Lemma 9 , we have

$$
\left\|u_{n}-p\right\|=\left\|T_{r_{n}} x_{n}-T_{r_{n}} p\right\| \leq\left\|x_{n}-p\right\|
$$

It follows from (25) that

$$
\begin{aligned}
& \left\|y_{n}-p\right\| \\
& \quad=\left\|\beta_{n}\left(x_{n}-p\right)+\left(1-\beta_{n}\right)\left(L_{n} u_{n}-L_{n} p\right)\right\| \\
& \quad \leq \beta_{n}\left\|x_{n}-p\right\|+\left(1-\beta_{n}\right)\left\|u_{n}-p\right\| \\
& \quad \leq\left\|x_{n}-p\right\| .
\end{aligned}
$$

Further we get

$$
\begin{aligned}
\| x_{n+1} & -p \| \\
= & \| \alpha_{n}\left(\gamma V x_{n}-\mu A p\right)+\left(I-\mu \alpha_{n} A\right) y_{n} \\
& -\left(I-\mu \alpha_{n} A\right) p \| \\
\leq & \alpha_{n}\left(\left\|\gamma V x_{n}-\gamma V p\right\|+\|\gamma V p-\mu A p\|\right) \\
& +\left(1-\alpha_{n} \tau\right)\left\|y_{n}-p\right\| \\
\leq & \alpha_{n} l \gamma\left\|x_{n}-p\right\|+\alpha_{n}\|\gamma V p-\mu A p\| \\
& +\left(1-\alpha_{n} \tau\right)\left\|y_{n}-p\right\| \\
= & \left(1-\alpha_{n}(\tau-l \gamma)\right)\left\|x_{n}-p\right\| \\
& +\alpha_{n}(\tau-l \gamma) \frac{\|\gamma V p-\mu A p\|}{\tau-l \gamma} \\
\leq & \max \left\{\left\|x_{n}-p\right\|, \frac{\|\gamma V p-\mu A p\|}{\tau-l \gamma}\right\} .
\end{aligned}
$$

By induction, we obtain $\left\|x_{n}-p\right\| \leq \max \left\{\left\|x_{1}-p\right\|, \| \gamma V p-\right.$ $\mu A p \| /(\tau-l \gamma)\}, n \geq 1$. Hence, $\left\{x_{n}\right\}$ is bounded, so are $\left\{u_{n}\right\}$ and $\left\{y_{n}\right\}$. It follows from the Lipschitz continuity of $A$ and $V$ that $\left\{A x_{n}\right\},\left\{A u_{n}\right\}$, and $\left\{V x_{n}\right\}$ are also bounded. From the nonexpansivity of $L_{n}$, it follows that $\left\{L_{n} x_{n}\right\}$ is also bounded.

Step 2. Show that

$$
\left\|x_{n+1}-x_{n}\right\| \longrightarrow 0
$$

Suppose $x_{n+1}=\beta_{n} x_{n}+\left(1-\beta_{n}\right) z_{n}$, then $z_{n}=\left(x_{n+1}-\beta_{n} x_{n}\right) /(1-$ $\left.\beta_{n}\right)=\left(\alpha_{n} \gamma V x_{n}+\left(I-\mu \alpha_{n} A\right) y_{n}-\beta_{n} x_{n}\right) /\left(1-\beta_{n}\right)$.

Hence, we have

$$
\begin{aligned}
z_{n+1} & -z_{n} \\
= & \frac{\alpha_{n+1} \gamma V x_{n+1}+\left(I-\mu \alpha_{n+1} A\right) y_{n+1}-\beta_{n+1} x_{n+1}}{1-\beta_{n+1}} \\
& -\frac{\alpha_{n} \gamma V x_{n}+\left(I-\mu \alpha_{n} A\right) y_{n}-\beta_{n} x_{n}}{1-\beta_{n}} \\
= & \frac{\alpha_{n+1}\left(\gamma V x_{n+1}-\mu A y_{n+1}\right)}{1-\beta_{n+1}}+\frac{y_{n+1}-\beta_{n+1} x_{n+1}}{1-\beta_{n+1}} \\
& -\frac{\alpha_{n}\left(\gamma V x_{n}-\mu A y_{n}\right)}{1-\beta_{n}}-\frac{y_{n}-\beta_{n} x_{n}}{1-\beta_{n}}
\end{aligned}
$$




$$
\begin{aligned}
= & \frac{\alpha_{n+1}\left(\gamma V x_{n+1}-\mu A y_{n+1}\right)}{1-\beta_{n+1}} \\
& +\frac{\beta_{n+1} x_{n+1}+\left(1-\beta_{n+1}\right) L_{n+1} u_{n+1}-\beta_{n+1} x_{n+1}}{1-\beta_{n+1}} \\
& -\frac{\alpha_{n}\left(\gamma V x_{n}-\mu A y_{n}\right)}{1-\beta_{n}}-\frac{\beta_{n} x_{n}+\left(1-\beta_{n}\right) L_{n} u_{n}-\beta_{n} x_{n}}{1-\beta_{n}} \\
\leq & \frac{\alpha_{n+1}\left(\gamma V x_{n+1}-\mu A y_{n+1}\right)}{1-\beta_{n+1}}-\frac{\alpha_{n}\left(\gamma V x_{n}-\mu A y_{n}\right)}{1-\beta_{n}} \\
& +L_{n+1} u_{n+1}-L_{n} u_{n} .
\end{aligned}
$$

Observe that

$$
\begin{aligned}
\left\|u_{n+1}-u_{n}\right\| & =\left\|T_{r_{n+1}} x_{n+1}-T_{r_{n}} x_{n}\right\| \\
& \leq\left\|T_{r_{n+1}} x_{n+1}-T_{r_{n+1}} x_{n}\right\|+\left\|T_{r_{n+1}} x_{n}-T_{r_{n}} x_{n}\right\| \\
& \leq\left\|x_{n+1}-x_{n}\right\|+\left\|T_{r_{n+1}} x_{n}-T_{r_{n}} x_{n}\right\| .
\end{aligned}
$$

By the definition of $L_{n}$, we have

$$
\begin{aligned}
& \left\|L_{n+1} u_{n+1}-L_{n} u_{n}\right\| \\
& =\left\|L_{n+1} u_{n+1}-L_{n+1} u_{n}\right\|+\left\|L_{n+1} u_{n}-L_{n} u_{n}\right\| \\
& \leq\left\|u_{n+1}-u_{n}\right\|+\left\|\sum_{i=1}^{n+1} \frac{\omega_{i}}{s_{n+1}} S_{i}^{\prime} u_{n}-\sum_{i=1}^{n} \frac{\omega_{i}}{s_{n}} S_{i}^{\prime} u_{n}\right\| \\
& \leq\left\|x_{n+1}-x_{n}\right\|+\left\|T_{r_{n+1}} x_{n}-T_{r_{n}} x_{n}\right\| \\
& \quad+\left\|\frac{\omega_{n+1}}{s_{n+1}} S_{n+1}^{\prime} u_{n}+\sum_{i=1}^{n}\left(\frac{\omega_{i}}{s_{n+1}}-\frac{\omega_{i}}{s_{n}}\right) S_{i}^{\prime} u_{n}\right\| \\
& \leq\left\|x_{n+1}-x_{n}\right\|+\left\|T_{r_{n+1}} x_{n}-T_{r_{n}} x_{n}\right\| \\
& \quad+\left\|\frac{\omega_{n+1}}{s_{n+1}} S_{n+1}^{\prime} u_{n}\right\|+\left\|\sum_{i=1}^{n} \frac{-\omega_{n+1} \omega_{i}}{s_{n+1} s_{n}} S_{i}^{\prime} u_{n}\right\| \\
& \leq\left\|x_{n+1}-x_{n}\right\|+\left\|T_{r_{n+1}} x_{n}-T_{r_{n}} x_{n}\right\|+2 M_{1} \frac{\omega_{n+1}}{s_{n+1}},
\end{aligned}
$$

where $M_{1}=\sup _{i, n \geq 1}\left\{\left\|S_{i}^{\prime} u_{n}\right\|\right\}$.

It follows from (30) and (32) that

$$
\begin{aligned}
\left\|z_{n+1}-z_{n}\right\| & \\
\leq & \frac{\alpha_{n+1}}{1-\beta_{n+1}}\left(\left\|\gamma V x_{n+1}\right\|+\left\|\mu A y_{n+1}\right\|\right) \\
& +\frac{\alpha_{n}}{1-\beta_{n}}\left(\left\|\gamma V x_{n}\right\|+\left\|\mu A y_{n}\right\|\right)+\left\|L_{n+1} u_{n+1}-L_{n} u_{n}\right\| \\
\leq & \left(\frac{\alpha_{n+1}}{1-\beta_{n+1}}+\frac{\alpha_{n}}{1-\beta_{n}}\right) M_{2}+\left\|x_{n+1}-x_{n}\right\| \\
\quad & \left\|T_{r_{n+1}} x_{n}-T_{r_{n}} x_{n}\right\|+2 M_{1} \frac{\omega_{n+1}}{s_{n+1}}
\end{aligned}
$$

where $M_{2}=\sup _{n}\left\{\left\|\gamma V x_{n}\right\|+\left\|\mu A y_{n}\right\|\right\}$.
Hence we get $\left\|z_{n+1}-z_{n}\right\|-\left\|x_{n+1}-x_{n}\right\| \leq\left\|T_{r_{n+1}} x_{n}-T_{r_{n}} x_{n}\right\|+$ $\left(\left(\alpha_{n+1} /\left(1-\beta_{n+1}\right)\right)+\left(\alpha_{n} /\left(1-\beta_{n}\right)\right)\right) M_{2}+M_{1}\left(\omega_{n+1} / S_{n+1}\right)$. Since $\sum_{n=1}^{\infty} \omega_{n}=1$ is convergent, it is easy to see that $\sum_{n=1}^{\infty} \omega_{n} / s_{n}$ is also convergent. Thus we have $\omega_{n} / s_{n} \rightarrow 0(n \rightarrow \infty)$.

From conditions (i) and (iii) and Lemma 11, we obtain

$$
\limsup _{n \rightarrow \infty}\left(\left\|z_{n+1}-z_{n}\right\|-\left\|x_{n+1}-x_{n}\right\|\right) \leq 0 .
$$

By Lemma 10, we have $\lim _{n \rightarrow \infty}\left\|z_{n}-x_{n}\right\|=0$. Thus

$$
\lim _{n \rightarrow \infty}\left\|x_{n+1}-x_{n}\right\|=\lim _{n \rightarrow \infty}\left(1-\beta_{n}\right)\left\|z_{n}-x_{n}\right\|=0 .
$$

By Lemma 11 and (30) and (29), we obtain

$$
\left\|u_{n+1}-u_{n}\right\| \longrightarrow 0
$$

Step 3. Show that

$$
\left\|x_{n}-L x_{n}\right\| \longrightarrow 0,
$$

where $L=\sum_{i=1}^{\infty} \omega_{i} S_{i}^{\prime}(i=1,2, \ldots)$.

Observe that

$$
\begin{aligned}
\left\|x_{n}-L_{n} x_{n}\right\| \leq & \left\|x_{n}-L_{n} u_{n}\right\|+\left\|L_{n} u_{n}-L_{n} x_{n}\right\| \\
\leq & \left\|x_{n}-L_{n} u_{n}\right\|+\left\|u_{n}-x_{n}\right\|, \\
\left\|x_{n}-L_{n} u_{n}\right\| \leq & \left\|x_{n}-x_{n+1}\right\|+\left\|x_{n+1}-y_{n}\right\|+\left\|y_{n}-L_{n} u_{n}\right\| \\
= & \left\|x_{n}-x_{n+1}\right\|+\alpha_{n}\left\|\gamma V x_{n}-\mu A y_{n}\right\| \\
& +\beta_{n}\left\|x_{n}-L_{n} u_{n}\right\| .
\end{aligned}
$$

From condition (i) and (25), we can obtain

$$
\begin{aligned}
& \left(1-\beta_{n}\right)\left\|x_{n}-L_{n} u_{n}\right\| \\
& \quad \leq\left\|x_{n}-x_{n+1}\right\|+\alpha_{n}\left\|\gamma V x_{n}-\mu A y_{n}\right\| \longrightarrow 0 .
\end{aligned}
$$

It follows from condition (ii) that

$$
\left\|x_{n}-L_{n} u_{n}\right\| \longrightarrow 0 .
$$

By Lemma 9, we get

$$
\begin{aligned}
\left\|u_{n}-p\right\|^{2} & =\left\|T_{r_{n}} x_{n}-T_{r_{n}} p\right\|^{2} \\
& \leq\left\langle T_{r_{n}} x_{n}-T_{r_{n}} p, x_{n}-p\right\rangle \\
& =\frac{1}{2}\left(\left\|u_{n}-p\right\|^{2}+\left\|x_{n}-p\right\|^{2}+\left\|x_{n}-u_{n}\right\|^{2}\right) .
\end{aligned}
$$

This implies that

$$
\left\|u_{n}-p\right\|^{2} \leq\left\|x_{n}-p\right\|^{2}-\left\|x_{n}-u_{n}\right\|^{2} .
$$

By nonexpansivity of $L_{n}$, we have

$$
\begin{aligned}
\left\|y_{n}-p\right\|^{2} & \leq \beta_{n}\left\|x_{n}-p\right\|^{2}+\left(1-\beta_{n}\right)\left\|u_{n}-p\right\|^{2} \\
& \leq\left\|x_{n}-p\right\|^{2}-\left(1-\beta_{n}\right)\left\|x_{n}-u_{n}\right\|^{2} .
\end{aligned}
$$


It follows from (25) that

$$
\begin{aligned}
\| x_{n+1}- & p \|^{2} \\
= & \| \alpha_{n}\left(\gamma V x_{n}-p\right)+\left(I-\mu \alpha_{n} A\right) y_{n}-\left(I-\mu \alpha_{n} A\right) p \\
& +\alpha_{n}(p-\mu A p) \|^{2} \\
\leq & \alpha_{n}\left\|\gamma V x_{n}-p\right\|^{2}+\left(1-\alpha_{n} \tau\right)\left\|y_{n}-p\right\|^{2} \\
& +\alpha_{n}\|p-\mu A p\|^{2} \\
\leq & \alpha_{n}\left\|\gamma V x_{n}-p\right\|^{2} \\
& +\left(1-\alpha_{n} \tau\right)\left(\left\|x_{n}-p\right\|^{2}-\left(1-\beta_{n}\right)\left\|x_{n}-u_{n}\right\|^{2}\right) \\
& +\alpha_{n}\|p-\mu A p\|^{2} \\
\leq & \alpha_{n}\left\|\gamma V x_{n}-p\right\|^{2}+\left\|x_{n}-p\right\|^{2} \\
& -\left(1-\beta_{n}\right)\left\|x_{n}-u_{n}\right\|^{2}+\alpha_{n}\|p-\mu A p\|^{2} .
\end{aligned}
$$

This implies that

$$
\begin{aligned}
&\left(1-\beta_{n}\right)\left\|x_{n}-u_{n}\right\|^{2} \\
& \leq \alpha_{n}\left(\left\|\gamma V x_{n}-p\right\|^{2}+\|p-\mu A p\|^{2}\right) \\
&+\left\|x_{n}-p\right\|^{2}-\left\|x_{n+1}-p\right\|^{2} \\
& \leq \alpha_{n}\left(\left\|\gamma V x_{n}-p\right\|^{2}+\|p-\mu A p\|^{2}\right) \\
&+\left(\left\|x_{n}-p\right\|+\left\|x_{n+1}-p\right\|\right)\left\|x_{n+1}-x_{n}\right\| .
\end{aligned}
$$

From conditions (i) and (ii) and (29), we have

$$
\left\|x_{n}-u_{n}\right\| \longrightarrow 0
$$

Thus, we get

$$
\left\|x_{n}-L_{n} x_{n}\right\| \longrightarrow 0
$$

On the other hand, we have

$$
\left\|x_{n}-L x_{n}\right\| \leq\left\|x_{n}-L_{n} x_{n}\right\|+\left\|L_{n} x_{n}-L x_{n}\right\| .
$$

Combining (47) and Lemma 7, we obtain (37).

Step 4. Show that

$$
\limsup _{n \rightarrow \infty}\left\langle(\gamma V-\mu A) x^{*}, x_{n}-x^{*}\right\rangle \leq 0,
$$

where $x^{*}=P_{\Omega}(I-\mu A+\gamma V) x^{*}$ is a unique solution of the variational inequality (24). Indeed, take a subsequence $\left\{x_{n_{j}}\right\}$ of $\left\{x_{n}\right\}$ such that

$$
\begin{aligned}
& \limsup _{n \rightarrow \infty}\left\langle(\gamma V-\mu A) x^{*}, x_{n}-x^{*}\right\rangle \\
& \quad=\lim _{j \rightarrow \infty}\left\langle(\gamma V-\mu A) x^{*}, x_{n_{j}}-x^{*}\right\rangle .
\end{aligned}
$$

Since $\left\{x_{n_{j}}\right\}$ is bounded, there exists a subsequence $\left\{x_{n_{j_{k}}}\right\}$ of $\left\{x_{n_{j}}\right\}$ which converges weakly to $q$. Without loss of generality, we can assume $x_{n_{j}} \rightarrow q$. From (37), we obtain $L x_{n_{j}} \rightarrow q$.

By the same argument as in the proof of Theorem 13, we have $q \in \Omega$. Since $x^{*}=P_{\Omega}(I-\mu A+\gamma V) x^{*}$, it follows that

$$
\begin{aligned}
& \limsup _{n \rightarrow \infty}\left\langle(\gamma V-\mu A) x^{*}, x_{n}-x^{*}\right\rangle \\
& \quad=\lim _{j \rightarrow \infty}\left\langle(\gamma V-\mu A) x^{*}, x_{n_{j}}-x^{*}\right\rangle \\
& =\left\langle(\gamma V-\mu A) x^{*}, q-x^{*}\right\rangle \leq 0 .
\end{aligned}
$$

Step 5. Show that

$$
x_{n} \longrightarrow x^{*} \text {. }
$$

Since

$$
\begin{aligned}
& \left\langle(\gamma V-\mu A) x^{*}, x_{n+1}-x^{*}\right\rangle \\
& \quad=\left\langle(\gamma V-\mu A) x^{*}, x_{n+1}-x_{n}\right\rangle+\left\langle(\gamma V-\mu A) x^{*}, x_{n}-x^{*}\right\rangle \\
& \leq\left\|(\gamma V-\mu A) x^{*}\right\|\left\|x_{n+1}-x_{n}\right\|+\left\langle(\gamma V-\mu A) x^{*}, x_{n}-x^{*}\right\rangle .
\end{aligned}
$$

It follows from (29) and (51) that

$$
\begin{aligned}
\limsup _{n \rightarrow \infty}\left\langle(\gamma V-\mu A) x^{*}, x_{n+1}-x^{*}\right\rangle \leq 0, \\
\left\|x_{n+1}-x^{*}\right\|^{2} \\
=\left\|\alpha_{n} \gamma V x_{n}+\left(I-\mu \alpha_{n} A\right) y_{n}-x^{*}\right\|^{2} \\
=\|\left(I-\mu \alpha_{n} A\right) y_{n}-\left(I-\mu \alpha_{n} A\right) x^{*} \\
\quad+\alpha_{n}\left(\gamma V x_{n}-\mu A x^{*}\right) \|^{2} \\
\leq\left\|\left(I-\mu \alpha_{n} A\right) y_{n}-\left(I-\mu \alpha_{n} A\right) x^{*}\right\|^{2} \\
+2 \alpha_{n}\left\langle\gamma V x_{n}-\mu A x^{*}, x_{n+1}-x^{*}\right\rangle \\
\leq\left(1-\alpha_{n} \tau\right)^{2}\left\|y_{n}-x^{*}\right\|^{2}+2 \alpha_{n}\left\langle\gamma V x_{n}-\gamma V x^{*}, x_{n+1}-x^{*}\right\rangle \\
+2 \alpha_{n}\left\langle(\gamma V-\mu A) x^{*}, x_{n+1}-x^{*}\right\rangle \\
\leq\left(1-\alpha_{n} \tau\right)^{2}\left\|x_{n}-x^{*}\right\|^{2} \\
+\alpha_{n} l \gamma\left(\left\|x_{n}-x^{*}\right\|^{2}+\left\|x_{n+1}-x^{*}\right\|^{2}\right) \\
+2 \alpha_{n}\left\langle(\gamma V-\mu A) x^{*}, x_{n+1}-x^{*}\right\rangle .
\end{aligned}
$$

This implies that

$$
\begin{aligned}
& \left\|x_{n+1}-x^{*}\right\|^{2} \\
& \quad \leq \frac{\left(1-\alpha_{n} \tau\right)^{2}+\alpha_{n} l \gamma}{1-\alpha_{n} l \gamma}\left\|x_{n}-x^{*}\right\|^{2}
\end{aligned}
$$


TABLE 1: $x_{1}=1 / 2$.

\begin{tabular}{lcc}
\hline$n$ (iterative number) & $x^{(1)}$ (initial guess) & Errors $(n)$ \\
\hline 94 & 0.9969 & $3.1 \times 10^{-3}$ \\
150 & 0.9981 & $1.9 \times 10^{-3}$ \\
450 & 0.9994 & $6.3 \times 10^{-4}$ \\
\hline
\end{tabular}

$$
\begin{aligned}
& \quad+\frac{2 \alpha_{n}}{1-\alpha_{n} l \gamma}\left\langle(\gamma V-\mu A) x^{*}, x_{n+1}-x^{*}\right\rangle \\
& \leq\left(1-\frac{2 \alpha_{n}(\tau-l \gamma)}{1-\alpha_{n} l \gamma}\right)\left\|x_{n}-x^{*}\right\|^{2} \\
& +\frac{2 \alpha_{n}}{1-\alpha_{n} l \gamma}\left\langle(\gamma V-\mu A) x^{*}, x_{n+1}-x^{*}\right\rangle \\
& +\frac{\left(\alpha_{n} \tau\right)^{2}}{1-\alpha_{n} l \gamma} M_{3},
\end{aligned}
$$

where $M_{3}=\sup _{n}\left\|x_{n}-x^{*}\right\|^{2}, n \geq 1$. Put $\gamma_{n}=2 \alpha_{n}(\tau-$ $l \gamma) /\left(1-\alpha_{n} l \gamma\right), \delta_{n}=\left(2 \alpha_{n} /\left(1-\alpha_{n} l \gamma\right)\right)\left\langle(\gamma V-\mu A) x^{*}\right.$, $\left.x_{n+1}-x^{*}\right\rangle+\left(\left(\alpha_{n} \tau\right)^{2} /\left(1-\alpha_{n} l \gamma\right)\right) M_{3}$. It is easy to see that $\lim \sup _{n \rightarrow \infty} \delta_{n} / \gamma_{n} \leq 0$. Hence, by Lemma 5 , the sequence $\left\{x_{n}\right\}$ converges strongly to $x^{*}$.

Remark 14. If we extend the equilibrium problem to be system of equilibrium problems, we still obtain the desired result by the similar proof of Theorem 13 .

\section{Numerical Result}

In this section, we consider the following two simple examples to demonstrate the effectiveness, realization, and convergence of the algorithm in Theorem 13. Further, we compare convergence rates of the algorithm in this paper and [15].

First, we give an example as follows.

Example 15. In Theorem 13, let $H=R, C=[1 / 4,+\infty), F \equiv 0$, for all $x, y \in C$. Define $S_{0}: x \mapsto \sqrt{x}, S_{1}: x \mapsto x+(\pi / 4)-$ $\operatorname{arctanx}$, and let $S_{n}=S_{\text {nmod2 }}, n=1,2, \ldots$. Take $A=I$ with Lipschitz constant $k=1$ and strongly monotone constant $\eta=$ $1, V x=2 x$, for all $x \in H$ with Lipschitz coefficient $l=2$. Give the parameters $\alpha_{n}=1 / 20 \sqrt{n}, \beta_{n}=1 / 4$ for every $n \geq 1$, and fix $\mu=1$ and $\gamma=1 / 8$. Then $\left\{x_{n}\right\}$ is the sequence generated by

$$
\begin{gathered}
y_{n}=\frac{1}{4} x_{n}+\frac{3}{4} L_{n} x_{n}, \\
x_{n+1}=\frac{1}{8} \frac{1}{20 \sqrt{n}} 2 x_{n}+\left(1-\frac{1}{20 \sqrt{n}}\right) y_{n} .
\end{gathered}
$$

As $n \rightarrow \infty$, we have $\left\{x_{n}\right\} \rightarrow x^{*}=1$.

Let $\omega_{i}=1 / 2^{i}, i=1,2, \ldots$; then we have $\sum_{i=1}^{\infty} \omega_{i}=1$. Take the initial guess $x_{1}=1 / 2$, using software MATLAB R2012, we obtain the numerical experiment results in Table 1.

Let $R^{2}$ be the two-dimensional Euclidean space with usual inner product $\left\langle x^{(1)}, x^{(2)}\right\rangle=x_{1}^{(1)} x_{1}^{(2)}+x_{2}^{(1)} x_{2}^{(2)} \quad\left(\right.$ for all $x^{(1)}=$
TABLE 2: (a) $x^{(1)}=(1,0)^{\top}$. (b) $x^{(1)}=(1,0)^{\top}$.

(a)

\begin{tabular}{lcc}
\hline$n$ (iterative number) & $x^{(1)}$ (initial guess) & Errors $(n)$ \\
\hline 10 & $(0.8289,0.5521)$ & $4.6 \times 10^{-3}$ \\
50 & $(0.8308,0.5559)$ & $3.8 \times 10^{-4}$ \\
100 & $(0.8310,0.5561)$ & $1.9 \times 10^{-4}$ \\
\hline
\end{tabular}

(b)

\begin{tabular}{lcc}
\hline$n$ (iterative number) & $x^{(1)}$ (initial guess) & Errors $(n)$ \\
\hline 10 & $(0.8271,0.5521)$ & $5.6 \times 10^{-3}$ \\
50 & $(0.8308,0.5558)$ & $4.7 \times 10^{-4}$ \\
100 & $(0.8309,0.5561)$ & $2.2 \times 10^{-4}$ \\
\hline
\end{tabular}

$\left.\left(x_{1}^{(1)}, x_{2}^{(1)}\right)^{\top}, x^{(2)}=\left(x_{1}^{(2)}, x_{2}^{(2)}\right)^{\top} \in R^{2}\right)$ and induced norm $\|x\|=\sqrt{x_{1}^{2}+x_{2}^{2}} \quad\left(\right.$ for all $\left.x=\left(x_{1}, x_{2}\right)^{\top} \in R^{2}\right)$.

Next, we consider another simple example.

Example 16. In Theorem 13, let $H=R^{2}, C=[0,1] \times$ $[0,1], F \equiv 0$, for all $x, y \in C$. Give $S_{1}=I, S_{0}: x=$ $\left(x_{1}, x_{2}\right)^{\top} \mapsto\left(\sin \left(\left(x_{1}+x_{2}\right) / \sqrt{2}\right), \cos \left(\left(x_{1}+x_{2}\right) / \sqrt{2}\right)\right)^{\top}$, and let $S_{n}=S_{\text {nmod2 }}, n=1,2, \ldots \omega_{n}=1 / 2^{n} \in(0,1), n \geq 1$. Take $A=I$ with Lipschitz constant $k=1$ and strongly monotone constant $\eta=1, f(x)=\left((1 / 4) x_{1},-(1 / 4) x_{2}\right)^{\top}$, for all $x \in H$ with contraction coefficient $\rho=1 / 4$. Give the parameters $\alpha_{n}=1 / 10 n, \beta_{n}=1 / 2$ for every $n \geq 1$, and fix $\mu=1$ and $\gamma=1$. Then $\left\{x^{(n)}\right\}$ is the sequence generated by

$$
\begin{gathered}
y^{(n)}=\frac{1}{2} x^{(n)}+\frac{1}{2} L_{n} x^{(n)}, \\
x^{(n+1)}=\frac{1}{10 n}\left(\frac{1}{4} x_{1}^{(n)},-\frac{1}{4} x_{2}^{(n)}\right)+\frac{10 n-1}{10 n} y^{(n)},
\end{gathered}
$$

As $n \rightarrow \infty$, we have $\left\{x^{(n)}\right\} \rightarrow x^{*} \approx(0.8310,0.5562)^{\top}$.

For analysis of the rate of convergence, we use the concept introduced by Rhoades [20] as follows.

Definition 17. Let $E$ be a closed interval on the real line and $f: E \rightarrow E$ a continuous function. Suppose that $\left\{x_{n}\right\}_{n=1}^{\infty}$ and $\left\{y_{n}\right\}_{n=1}^{\infty}$ are two iterations which converge to the fixed point $p$ of $f$. Then, $\left\{x_{n}\right\}_{n=1}^{\infty}$ is said to converge faster than $\left\{y_{n}\right\}_{n=1}^{\infty}$ if

$$
\left\|x_{n}-p\right\| \leq\left\|y_{n}-p\right\|, \quad \forall n \geq 1 .
$$

Now we turn to numerical simulation using the algorithm (57). Take the initial guess $x^{(1)}=(1,0)^{\top}$ and $x^{(1)}=(1,1)^{\top}$, respectively. All the numerical experiment results are given in Tables 2(a) and 3(a). Then we realize the algorithm in [15], and the $W$-mapping is used in the paper. Further we obtain the corresponding numerical results which can be found in Tables 2(b) and 3(b).

It is easy to see that the approximation values obtained by the algorithm (25) in this paper are more close to the common fixed point $x^{*}$ at the same iterative number. And from the computer programming point of view, the algorithm is easier to implement in this paper. 


$$
\text { TABle 3: (a) } x^{(1)}=(1,1)^{\top} \text {. (b) } x^{(1)}=(1,1)^{\top} \text {. }
$$

(a)

\begin{tabular}{lcc}
\hline$n$ (iterative number) & $x^{(1)}$ (initial guess) & Errors $(n)$ \\
\hline 10 & $(0.8341,0.5534)$ & $9.7 \times 10^{-4}$ \\
50 & $(0.8308,0.5559)$ & $3.8 \times 10^{-4}$ \\
100 & $(0.8310,0.5561)$ & $1.9 \times 10^{-4}$ \\
\hline
\end{tabular}

(b)

\begin{tabular}{lcl}
\hline$n$ (iterative number) & $x^{(1)}$ (initial guess) & Errors $(n)$ \\
\hline 10 & $(0.8359,0.5531)$ & $2.3 \times 10^{-3}$ \\
50 & $(0.8308,0.5558)$ & $4.7 \times 10^{-4}$ \\
100 & $(0.8309,0.5561)$ & $2.1 \times 10^{-4}$ \\
\hline
\end{tabular}

\section{Acknowledgments}

The author would like to thank the referee for valuable suggestions to improve the paper and the Fundamental Research Funds for the Central Universities (Grant ZXH2012K001).

\section{References}

[1] F. E. Browder and W. V. Petryshyn, "Construction of fixed points of nonlinear mappings in Hilbert space," Journal of Mathematical Analysis and Applications, vol. 20, pp. 197-228, 1967.

[2] E. Blum and W. Oettli, "From optimization and variational inequalities to equilibrium problems," The Mathematics Student, vol. 63, no. 1-4, pp. 123-145, 1994.

[3] S. Chang, H. W. Joseph Lee, and C. K. Chan, "A new method for solving equilibrium problem fixed point problem and variational inequality problem with application to optimization," Nonlinear Analysis: Theory, Methods \& Applications, vol. 70, no. 9, pp. 3307-3319, 2009.

[4] Y. Liu, "A general iterative method for equilibrium problems and strict pseudo-contractions in Hilbert spaces," Nonlinear Analysis: Theory, Methods \& Applications, vol. 71, no. 10, pp. 4852-4861, 2009.

[5] G. Marino and H.-K. Xu, "A general iterative method for nonexpansive mappings in Hilbert spaces," Journal of Mathematical Analysis and Applications, vol. 318, no. 1, pp. 43-52, 2006.

[6] S. Takahashi and W. Takahashi, "Strong convergence theorem for a generalized equilibrium problem and a nonexpansive mapping in a Hilbert space," Nonlinear Analysis: Theory, Methods \& Applications, vol. 69, no. 3, pp. 1025-1033, 2008.

[7] P. L. Combettes and S. A. Hirstoaga, "Equilibrium programming in Hilbert spaces," Journal of Nonlinear and Convex Analysis, vol. 6, no. 1, pp. 117-136, 2005.

[8] W. R. Mann, "Mean value methods in iteration," Proceedings of the American Mathematical Society, vol. 4, pp. 506-510, 1953.

[9] K. Shimoji and W. Takahashi, "Strong convergence to common fixed points of infinite nonexpansive mappings and applications," Taiwanese Journal of Mathematics, vol. 5, no. 2, pp. 387404, 2001

[10] G. L. Acedo and H.-K. Xu, "Iterative methods for strict pseudocontractions in Hilbert spaces," Nonlinear Analysis: Theory, Methods \& Applications, vol. 67, no. 7, pp. 2258-2271, 2007.
[11] M. Tian, "A general iterative algorithm for nonexpansive mappings in Hilbert spaces," Nonlinear Analysis: Theory, Methods \& Applications, vol. 73, no. 3, pp. 689-694, 2010.

[12] I. Yamada, "The hybrid steepest descent method for the variational inequality problem over the intersection of fixed point sets of nonexpansive mappings," in Inherently Parallel Algorithms in Feasibility and Optimization and Their Applications (Haifa, 2000), vol. 8 of Studies in Computational Mathematics, pp. 473-504, North-Holland, Amsterdam, The Netherlands, 2001.

[13] S. Wang, "A general iterative method for obtaining an infinite family of strictly pseudo-contractive mappings in Hilbert spaces," Applied Mathematics Letters, vol. 24, no. 6, pp. 901-907, 2011.

[14] S. N. He and W. W. Sun, "New hybrid steepest descent algorithms for variational inequalities over the common fixed points set of infinite nonexpansive mappings," WSEAS Transactions on Mathematics, vol. 11, pp. 83-92, 2012.

[15] P. Duan and A. Wang, "General iterative methods for equilibrium problems and infinitely many strict pseudocontractions in Hilbert spaces," Journal of Applied Mathematics, vol. 2012, Article ID 602513, 17 pages, 2012.

[16] H. K. Xu, "An iterative approach to quadratic optimization," Journal of Optimization Theory and Applications, vol. 116, no. 3, pp. 659-678, 2003.

[17] Y. Yao, M. A. Noor, and Y.-C. Liou, "On iterative methods for equilibrium problems," Nonlinear Analysis: Theory, Methods \& Applications, vol. 70, no. 1, pp. 497-509, 2009.

[18] L.-C. Ceng and J.-C. Yao, "Hybrid viscosity approximation schemes for equilibrium problems and fixed point problems of infinitely many nonexpansive mappings," Applied Mathematics and Computation, vol. 198, no. 2, pp. 729-741, 2008.

[19] T. Suzuki, "Strong convergence of Krasnoselskii and Mann's type sequences for one-parameter nonexpansive semigroups without Bochner integrals," Journal of Mathematical Analysis and Applications, vol. 305, no. 1, pp. 227-239, 2005.

[20] B. E. Rhoades, "Comments on two fixed point iteration methods," Journal of Mathematical Analysis and Applications, vol. 56, no. 3, pp. 741-750, 1976. 


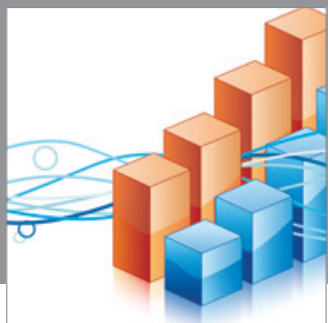

Advances in

Operations Research

mansans

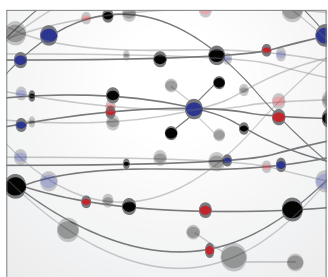

The Scientific World Journal
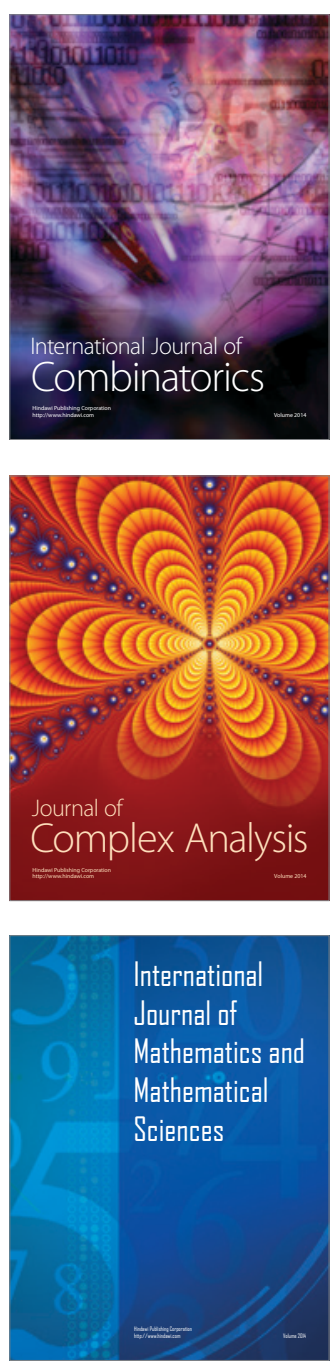
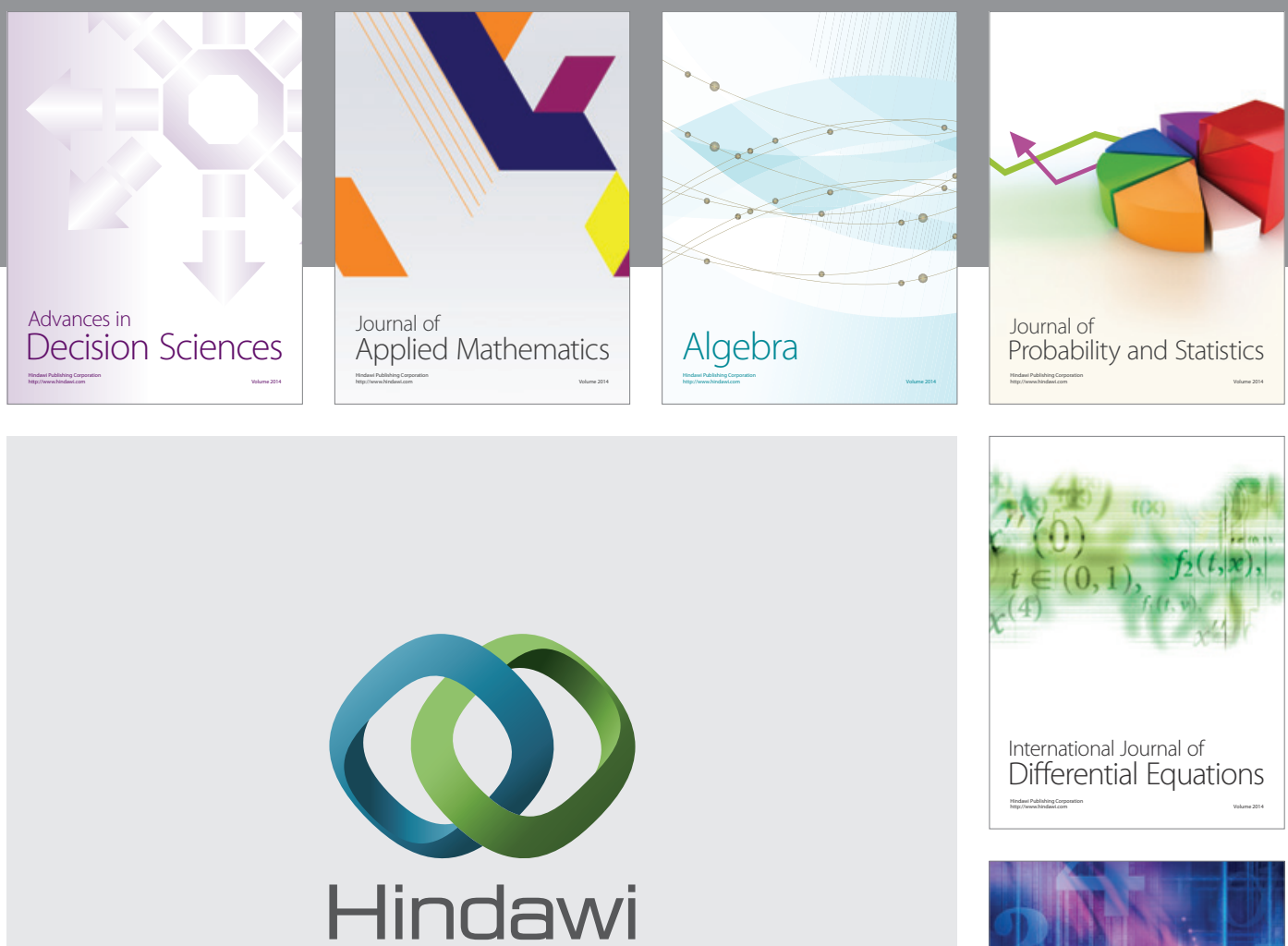

Submit your manuscripts at http://www.hindawi.com
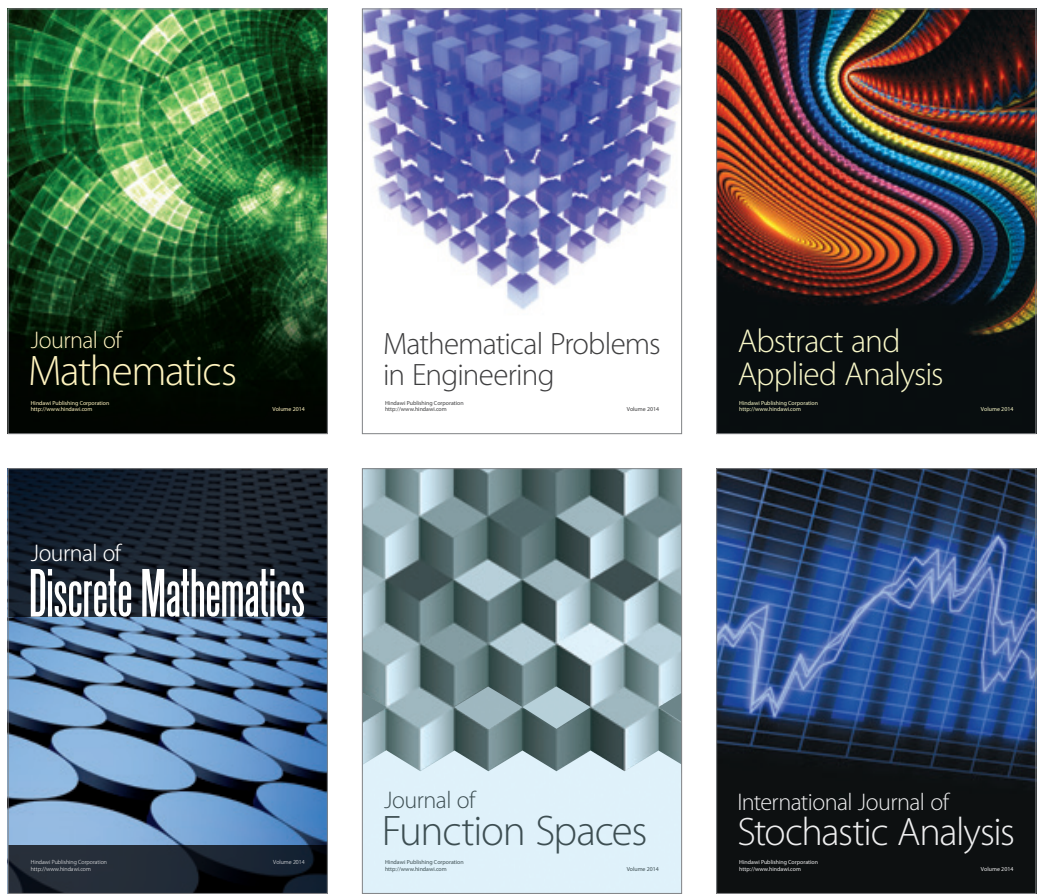

Journal of

Function Spaces

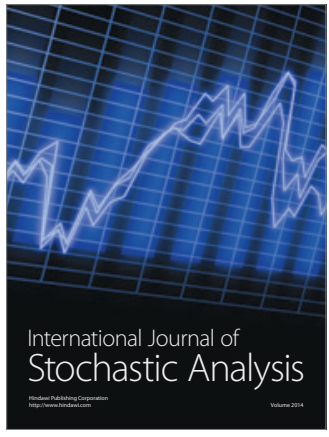

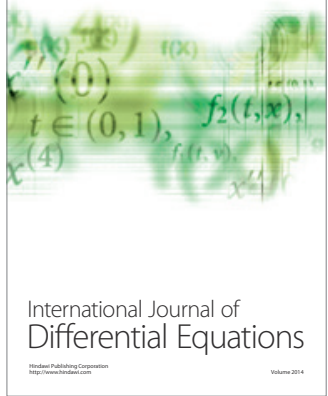
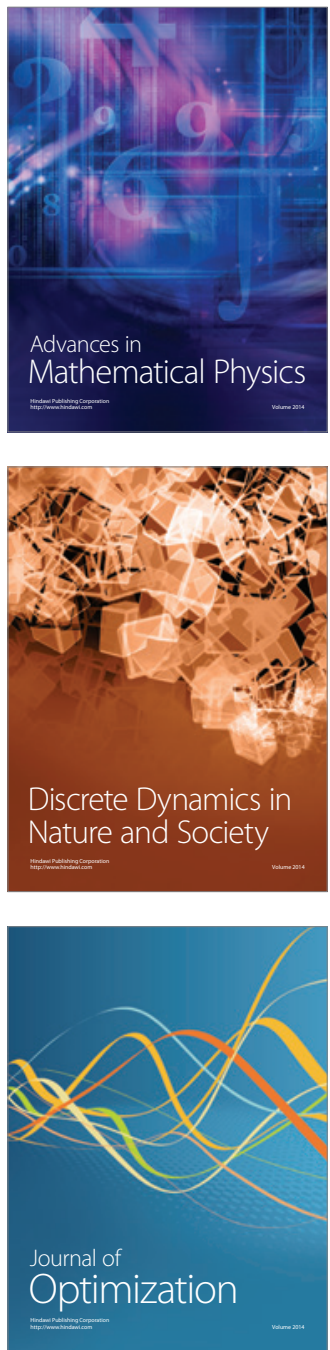